\title{
An Integrated Approach for Management of Activities, Documents and Evaluation of the Learning Process with a Mobile Component
}

\author{
Cosmina Ivan \\ Department of Computer Science \\ Technical University of Cluj-Napoca \\ Cluj County, Romania
}

\author{
Tudor- Adrian Vlas \\ MSc Student in Software Engineering \\ Department of Computer Science \\ Technical University of Cluj-Napoca \\ Cluj County, Romania
}

\begin{abstract}
In the past years the technology took a big turn and started to enter almost any common field of interest and activity. Motivated by this, a lot of applications were developed to overcome the time issue, in order to be able to decrease the amount of time involved using traditional work style. In this paper the design and implementation of The School Information Management System (SIMS). The main idea of the system, was to offer support for an improved communication between teachers and parents and to help teachers and headmasters to manage their daily administrative tasks. Complementary, the evaluation of the students and teachers is achievable using the system, and the management of various activities is also possible using specific features of the system. By offering a smart UI, the users are able to resolve various tasks in a time - effective way. The system is structured in a web application and a mobile application, both sharing a common data storage. SIMS was designed as a distributed system and relays on the Java EE - the Enterprise solution offered by Sun, also the mobile application developed in Android was designed and developed with the main purpose to offer access-everywhere and every time to stored data of interest for parents from the educational process: student grades and absences .
\end{abstract}

\section{General Terms}

Distributed Systems, Information System, Web application, Mobile application

\section{Keywords}

School Information Management System (SIMS), Document and activities management, Learning process

\section{INTRODUCTION}

In every domain, the efficiency is the key, the reduction of the time needed to finish tasks and because of this a lot of activities were transferred and offered support in general or specific Information Systems from the education domain. The new technology era imposes the realization of Information systems that are offering more performance and security. The reduction of the time needed for the realization of tasks and also the costs involved were the main principles behind the transfer of the multiple activities in the virtual space.

\section{MOTIVATION}

The necessary resources involved in various activities for school management using traditional methods experienced a continuous growing, in terms of human resources needed and time involved and the idea of structuring all these activities, documents and also their administration part in an appropriate and as complete as possible Information system was revealed.
Initially reviewing the representative systems used by the schools in Romania and after searching for different solutions used by American colleges and middle level schools, it was found that most systems offer an online catalog with grades and only few of them integrate also some basic tasks necessary for administrative activities complementary to the learning process. Some of these systems treat differentially system stakeholders (as teachers, parents, students) based on their roles, but none of them offer a complete role list, necessary for an actual, comprehensive School Information system.

The need for a more complex and complete system is impending, integrating not only a smart school catalog, but starting to take into consideration the various roles and their activities specific to the learning process. An essential part of the educational process is based on various types of specific documents: for example, educational planning for the classes, the school timetable, or the school ledger.

The implementation of such a management system offers a lot of advantages for all user roles. .For example, a principle can benefit from a quick access to resource allocation, like assigning teachers to classes, assigning class masters to classes, etc.

For teachers is very easy to be able to add new grades to students or to notes absentees and also to create educational planning for classes that they teach.

The parents are provided with a smart way of gaining access to the data of interest, for example the grades of their children, the absences and they also can communicate with the class master and with the committee of parents.

Based on the idea of collecting in a most appropriate way the system requirements, after taking interviews to parents and also to a group of teachers, the necessity of such an application was revealed; also the mobile application was considered of great use by the parents.

\section{SYSTEM DESIGN GOALS}

The main objective of the system was to increase efficiency of various roles activities by using a consistent but also a simple GUI for the application. One of the main objective of the project we have decided, was that the system must support further development based on various real user feedback, after a production period of time, so it must be extensible.

The system must be secured taking into consideration the navigation inside the application and also the security of the sensitive data that users provide. The high number of types of users that are involved in the learning process revealed the 
decision to offer a solution, so the access to different types of resources was necessary.

The system needed to offer support for creating and administering documents of everyday use for various users, as educational planning, school ledger and the schedule of classes. The management of activities needed to include a schedule of all the known events and also meetings of different councils that the user may be part of, parent council meetings, teacher council meetings, and department meetings. In various situations, where there is a lack of communication between teachers and parents that needed to be addressed, the solution of using a forum like communication was designed and implemented to solve this issue.

Managing the evaluation doesn't just relay on the evaluation of students and grading them, the teachers are also evaluated by the school principal and the application also addresses these issues as well.

At the end of middle school a lot of students find difficult to decide between the options to choose for high school and the same happens with the students that finish high school and also can't decide what they want to study further on the line. The application offers them some sort of recommendation based on the results they gathered during middle school and/or high school.

\section{TECHNOLOGIES BACKGROUND}

The mobile application was developed based on recent studies which point out that a lot of users prefer using mobile applications instead of using browsers on mobile devices [3]. The article provides also the main motivation of this aspect and the main arguments are the easy of use and simplicity in development that a mobile application provides. Mobile application tend to have great impact on users because of the great experience it can offer by using different features that the platform provides, like push notifications or even using a smart authentication based on face recognition.

\subsection{Web Application Technologies}

The main technologies used for the development of the web application rely on the Java Enterprise Edition Platform, that differentiates from Java Standard Edition by offering developers the opportunity of creating modular and distributed applications. More details about the platform can be found in [16]

Enterprise Java Beans is a standard included in the Java EE's API which offers persistence using Java Persistence API, process of transactions, manage concurrency, events using JMS and also exposing business methods as web services [15].

There are three types of Java Beans: Session, Message-driven and Entity Beans and the first two are used for implementing the logic part of the application and the third one, Entity, ensures persistence.

\subsubsection{Session Beans}

Session Beans were used in the development with the @Local annotation for the simple reason that they get to be invoked by managed beans (Java Server Faces backend component) that run in the same Java Virtual Machine. Also the Session Beans have a stateless character, because they do not contain necessary data for the client or they execute a job for all the clients [2].

\subsubsection{Message Driven Beans}

Message driven beans are EJB components that are used for asynchronous communication between parts of the system. They work similar to a JMS message listener. More details about message driven beans can be found in [9]. In SIMS system, message driven beans are used for the asynchronous communication when the application needs to send email to users and the solution was adopted because the system could get stuck if the list of users would be very large and the system can freeze until all the emails would be sent.

\subsubsection{Java Server Faces - JSF}

Java Server Faces [12] is an open-source technology that helps in the creation of the GUI for the application. It is designed based on the Model-View-Controller pattern and it separates the construction of the interface from the business. It has been decided to use JSF because of all the advantages it offers, which include using the tags provided by an older standard, JSP, offering the opportunity of creating own components and the smart separation of the business logic.

\subsubsection{Prime Faces}

Along with JSF, it has been decided to use Prime Faces which is a framework that is used on top of JSF and which offers a lot of components of interest like a schedule, a lot of exportable formats and also supports better responsive access to pages on mobile devices [14]. The alternatives to Prime Faces are Ice Faces or Rich Faces, but recent studies demonstrates that a lot of clients prefer Prime Faces [10]. More details about how Prime Faces work and also the components that the framework offers can be found in [13].

\subsubsection{Database}

The application uses as a relational SQL Server database and not only the web application but also the mobile one are accessing the same shared database .The concurrent access and consistency of the data issues is taken care by the SGBD.

\subsubsection{Machine Learning}

Data mining or machine learning is generally used for extracting useful data from a knowledge base. Data mining techniques are useful for prediction. Classification is one of the techniques used by data mining and more details about how this works can be found in [11]. There are a lot of types of classifications like Bayes networks, neuronal networks, etc. [7]. Classifiers that use decision trees got very popular. $\mathrm{C} 4.5 / \mathrm{J} 4.8$ is one of the most used and popular one and it basically divides recursively a problem into small subproblems using divide and conquer as a basis [4], [8]. Weka [14] is a toolkit that offers a lot of learning algorithms that can be called within the java code. It works with .arff data files and it needs a base file with data to learn from and after that, using the desired algorithm you can get various types of predictions.

\subsubsection{Security}

The security of the sensitive data, like passwords, is a must in almost any application and also in web applications, and the solution was Salted Password Hashing [18]. The decision was made based on a set of criteria, the most important was that the chosen solution must be able to stop the dictionary attacks, which cannot be cracked using intensive GPU processing, in paper [17] are presented more details about this method. 


\subsection{Mobile Application Technologies}

\subsubsection{Web Services}

The main technologies used for developing the mobile application rely on web services, specifically on SOAP because of the increased security that provide. Simple Object Access Protocol reduce exchange of messages between the client and the server, messages use XML based protocols.

Web services usually are preferred because of the great flexibility they provide and also because of the extended use. They are not technology dependent and they are not specifically used for one programming language. The resources from [5] and [6] provide documentation on how to consume the SOAP web services and how a service is exposed and described by WSDL (Web Service Description Language).We choose JAX-WS as the API provided by Java Enterprise Edition, and used to create web services in the SOAP standard.

\subsubsection{Android Platform}

Android is a software platform and also an operating system for mobile devices, based on Linux core. They were first developed by Google and after by Open Handset Alliance.

The Android platform allows developers to use Java as the developing language and they also offer a lot of great libraries. The fact that Android is an open-source platform it is a great opportunity for developers to extend the existing functionalities and get them incorporated into the Core [1].

KSOAP2 is a library for Android that allows the easy consuming of SOAP web services and we have decided to integrate it in our implementation.

\section{RELATED WORK}

The related products that we have found as currently in use in educational institutions focus on managing the online grading and making these grades available for the parents. There are also solutions that take the problem a step forward by adding different types of functionality for other roles besides the teachers and parents, by taking into consideration the students also.

The study of related products was limited to the best three locally developed systems and one solution with large acceptance in various American middle level educational units and colleges. The three systems developed locally that were studied are catalog-scolar.ro, complement.ro and eracovita.ro:8033/catalog-online, and the forth analyzed system was

\section{Ekattor School Management System Pro [19].}

Even though some systems include the main roles of the users that may use the application, none of them take into consideration the sub-roles that may exist, like for example : head of the department, members in different councils, and also the possibility of letting the others to choose a specific role as for example a representative.
It has been found that all systems provide a basic login and administration of the account, with small variations regarding the offered data that is stored for everyone (profile pictures, mobile phone, email, etc.). Only complement.ro provides the management of a schedule for classes.

The system offers also support for the principle to manage the various education standards in terms of documents and task allocation, as for example it offers the functionality to evaluate the job of the teachers.

Both systems, Complement.ro and eracovita.ro:8033/catalog-online, provides functionality regarding graphical analysis of grades or attendances at class level or school level and also offer the possibility to export these into different types of formats (pdf, excel, csv). Complement.ro provides the basic functionality for the communication between the class master and the students and parents. Catalog-scolar doesn't take into consideration the possibility of motivating absences. The parents get notified when the student is graded by an email only in the complement.ro application

One great idea that the system e-racovita.ro:8033/catalogonline implements, is the import of grades from an excel file and this can be very practical if we take for example a possible small downtime of the system and then getting the system back up and running with all the necessary grades being up to date. In our solution both teachers and students have the possibility of downloading in pdf format the schedule of classes, also the parents can have access to the student's version.

The principal can assign teachers to classes and also can administer the head of each department, the administration of the class masters is also supported within the application, and quick access for all the users to lists with contact data of parents, teachers, students. Various documents as for example the school regulation can be downloaded in pdf format and the construction of educational planning or the management of the school ledger are also available within the application.

The profile prediction for students it's a very useful component for these kind of systems, as the possibility to offer students some guidance at the end of an educational level. We have found that some systems offer a mobile application, but none of them implement the important functionality which is a school notebook of grades.

In Table 1 contains the main similarities and differences between the related products that were studied and put into antithesis the functionalities offered by all of them.
(1) - e-racovita.ro:8033/catalog-online
(2) - catalog-scolar.ro
(3) - complement.ro
(4) - Ekattor School Management System Pro
(5) - Our System 
Table 1. Related systems comparison

\begin{tabular}{|c|c|c|c|c|c|}
\hline Role/Functionality & (1) & (2) & (3) & (4) & (5) \\
\hline $\begin{array}{l}\text { Admin, Teacher and Parent } \\
\text { Role }\end{array}$ & $\mathrm{X}$ & $\mathrm{X}$ & $\mathrm{X}$ & $\mathrm{X}$ & $\mathrm{X}$ \\
\hline $\begin{array}{l}\text { Principle and Class Master } \\
\text { Role }\end{array}$ & & $\mathrm{X}$ & $\mathrm{X}$ & $\mathrm{X}$ & $\mathrm{X}$ \\
\hline Student Role & $\mathrm{X}$ & & $\mathrm{X}$ & $\mathrm{X}$ & $\mathrm{X}$ \\
\hline $\begin{array}{l}\text { Subroles (ex. Head of } \\
\text { department, board members) }\end{array}$ & & & & & $\mathrm{X}$ \\
\hline $\begin{array}{l}\text { Edit own account and } \\
\text { password recovery }\end{array}$ & $\mathrm{X}$ & $\mathrm{X}$ & $\mathrm{X}$ & $\mathrm{X}$ & $\mathrm{X}$ \\
\hline Contact & $\mathrm{X}$ & $\mathrm{X}$ & $\mathrm{X}$ & $\mathrm{X}$ & $\mathrm{X}$ \\
\hline Calendar & & & $\mathrm{X}$ & $X$ & $\mathrm{X}$ \\
\hline Manage Teacher's accounts & & & $\mathrm{X}$ & $\mathrm{X}$ & $\mathrm{X}$ \\
\hline Allocation of teachers & & & & & $\mathrm{X}$ \\
\hline $\begin{array}{l}\text { Grades and absences reports } \\
+ \text { export PDF, CSV, Excel }+ \\
\text { graphics }\end{array}$ & $\mathrm{X}$ & & $\mathrm{X}$ & & $\mathrm{X}$ \\
\hline $\begin{array}{l}\text { Students timetable + } \\
\text { download PDF }\end{array}$ & & $\mathrm{X}$ & $\mathrm{X}$ & $\mathrm{X}$ & $\mathrm{X}$ \\
\hline $\begin{array}{l}\text { Open discussions: class } \\
\text { master-students, class } \\
\text { master - parents }\end{array}$ & & & $\mathrm{X}$ & & $\mathrm{X}$ \\
\hline Motivation absences & $\mathrm{X}$ & & $\mathrm{X}$ & $\mathrm{X}$ & $\mathrm{X}$ \\
\hline $\begin{array}{l}\text { Notify parents for new grade } \\
\text { or absence }\end{array}$ & & & $\mathrm{X}$ & & $\mathrm{X}$ \\
\hline Mobile Application & $\mathrm{X}$ & & & & $\mathrm{X}$ \\
\hline
\end{tabular}

\section{SYSTEM ARCHITECTURE}

\subsection{Web Application Architecture}

The architecture of the web application follows the three layer model, as seen Figure 1. The client interacts with the second level using a standard protocol like API (as for example RPC - remote procedure call). The middle layer interacts with the database server using standard protocols offered by the JDBC drivers that implement the defined interfaces in the JDBCAPI. This middle-tier contains most of the logic of the application, offering response to every client request. The three-tier model is a client-server architectural model where the user interface, the business logic and the database are separated and communicate vertically.

The presentation level offer information to users, gather information from the user and will send the gathered information to the business layer, and after the specific business logic is made, data is persisted into the database using the persistence layer. The business tier includes the following packages that were named to suggest their meaning.
The package design can be seen in Figure 1, and the naming convention points out the main components and the relationships between them.

The Managed Beans are the ones that are in between the first two layers and they actually make the connection between the user interface and the business logic. Managed beans are also called backing beans for Java Server Faces, beans that contain some business logic but usually the small validations and verifications of the gathered data from the user, to prevent sending data that is not in the right format and also prevent sql injection.

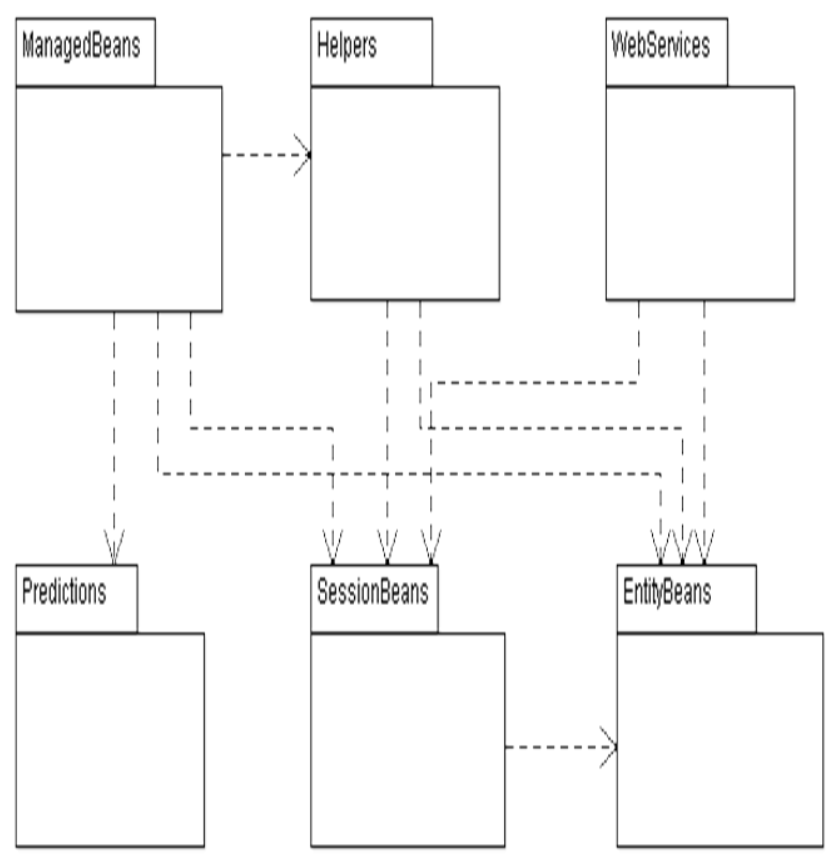

Fig 1: Package design

The entity beans are classes that map the database tables oneon-one. They get to be generated based on the existing structure into the database. The attributes and all the basic CRUD methods are also provided.

Session beans are the local interfaces used, and as previously mentioned, because these run on the same java virtual machine, they get to be annotated with @ Local. Each entity bean get to have an interface and also a class that implements that interface.

Message Driven Beans are the beans used to help with the asynchronous processing when sending emails. The actual communication is called the point-to-point communication and involves using a queue that resides on the server and where all the messages are sent. Each time a message is processed the next will be taken from the queue, it respects the first-in-first-out principle. 


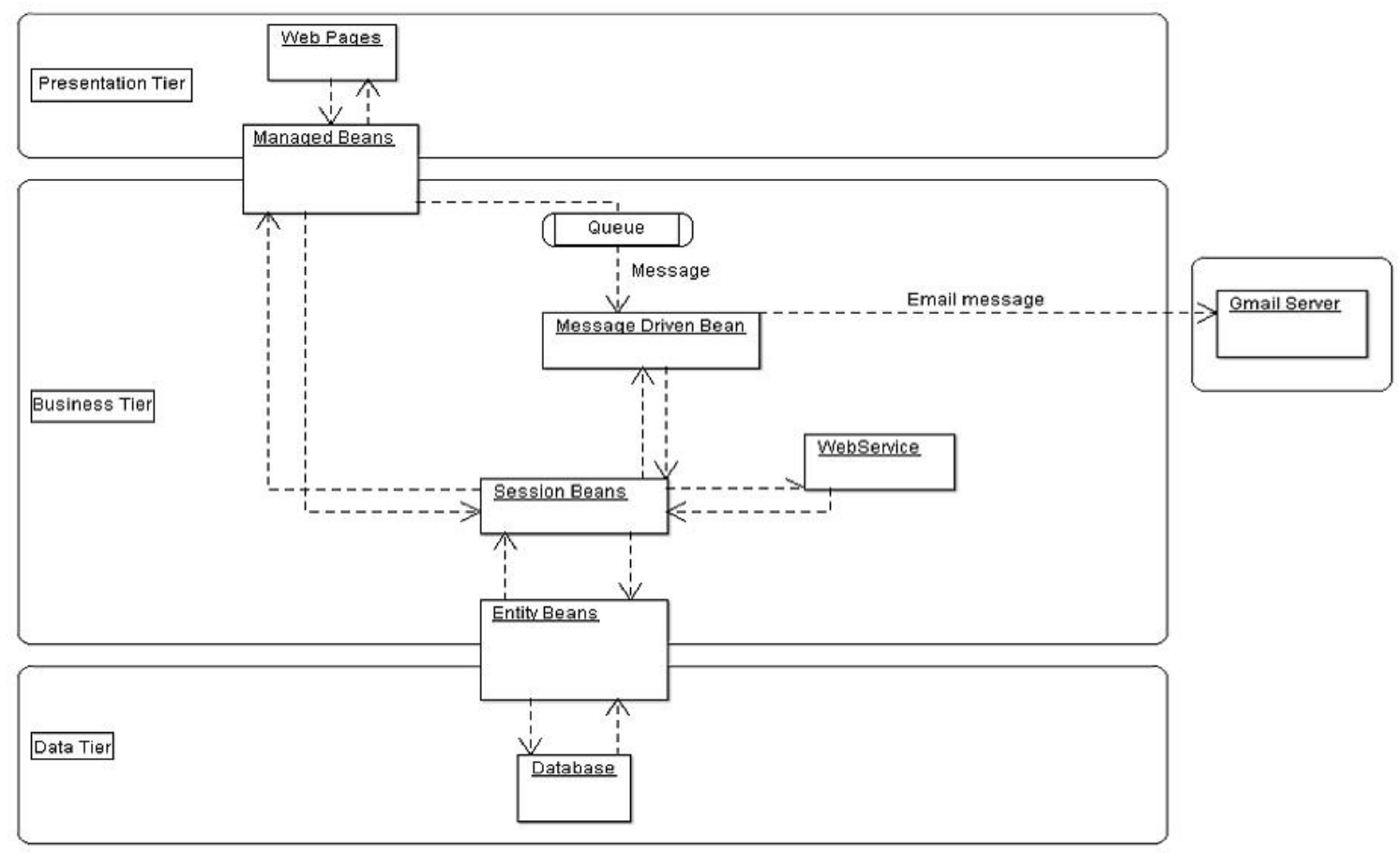

Fig 2: Web application Architectural Schema

\subsection{Web - Mobile Applications Communication}

The communication between the mobile application and the web server application is made using the SOAP web service as also described by Figure 3.

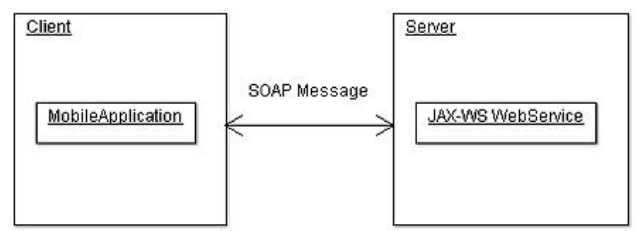

Fig 3: Web-Mobile SOAP communication

\subsection{Web Application - flow of possible options}

To present in a more intuitive way, the functionalities available for the system, for various user roles, are presented in a cumulative and complex use case diagram in Figure 4. In this figure, was point out how the subroles: head of department, class master and also board member have specific functionalities but also inherit from their parent role, the teacher. In real world scenarios, a user can play more than single role form the use case diagram. 


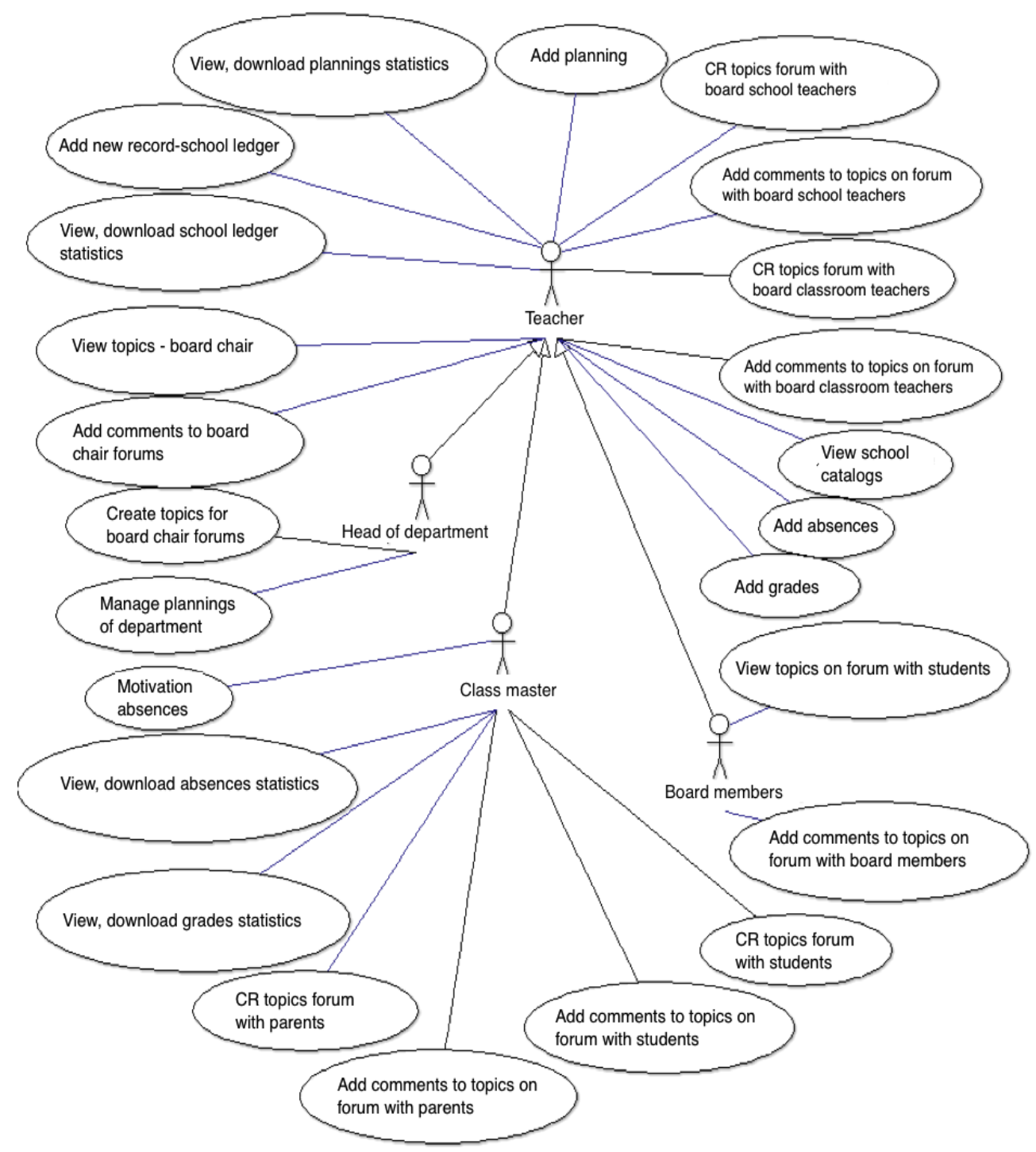

Fig 4: Teacher user role and subroles

\subsection{Data Vulnerabilities}

The vulnerability of the data is taken care of by using the Entity Manager provided by Java EE. Entity manager is an interface used to delete or add any transaction that implies the persistence of the data. The Persistence Unit is the set of types of entities that can get to be managed by the Entity Manager. By using the EM it's possible to create queries using the createNativeQuery where you can set parameters using setParameter and by using this standard the system is not exposed to any damage that may occur from malicious provided data.

\subsection{Mobile Application Architecture}

The mobile's application architecture was designed for Android using Java and using the Android SDK and also for intermediary version testing was done using the Genymotion emulator [20].

Figure 5 covers the adopted model of design used for the mobile application. In Android a new interface page is called an Activity, which besides the typical layout has a backing class that takes care of the events that may occur at the interface level.

The mobile application was created only for two types of users, for parents and also for students. The application may very well replace the grades notebook that is used at this point Not only the grades get to be seen within the application, also the absences are presented to parents. Also, at this point, whenever a new grade or absence is added, the parent is notified with an email. 


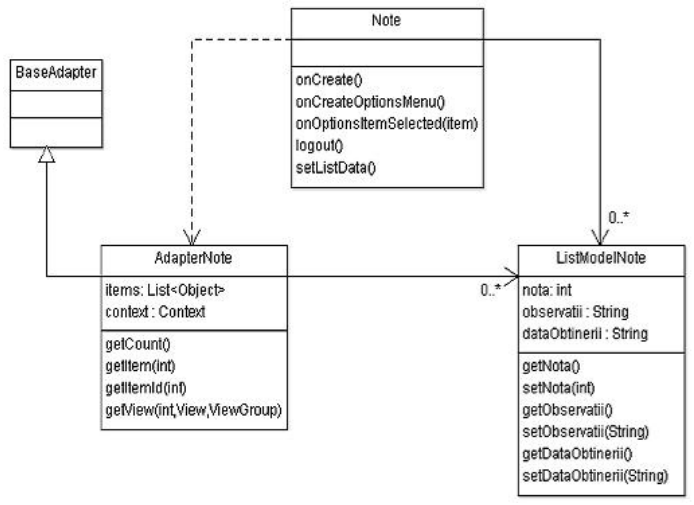

Fig 5: Mobile Application Architecture Design

\subsection{Deployment Schema}

The deployment is straightforward for both applications as is presented in Figure 6.

\subsection{Data persistence}

The database respects the conventions for it to be in Normal Boyce Code form by not having any functional dependencies than the ones determined by the primary key. For the whole system there was necessary to use 49 tables organized in a relational model.

\section{TESTING}

The development of the system was iterative and by that, the testing of new functionalities took place after the component was finished with the development. Most of the components were manually tested by following basic scenarios and continuing with trying to provide the system with malicious data and see how it reacts.

Besides the manual testing of the system, we created a Google Form that contained some questions regarding finding some places within the application and also to test some actual functionality, like for example adding a new grade or filling the school ledger. The user testing was made during a work day at a local high school. The teachers were not trained and they did not get any preliminary course about how to use the application. They were presented with the login screen of the application and they were provided with the credentials to be able to login and do the asked questions.

The overall results for the test was very successful and a lot of teachers considered the application to be very intuitive and they highlighted that using the application they could gain more time for preparing the classes and could have access to everything in one place. Also, the mobile application was considered to be a good idea because the parents are informed in real time about their children performance and presence.

The prediction component of the system was considered to be very useful and the continuous improvement of the results it provides was considered to be a great idea.

\section{FURTHER DEVELOPMENT}

The software development process is a continuous process with constant space for improvement, to be able to offer users new functionalities, and our system is not an exception to this rule. According to [1] ,from which result that the mobile takes over the web for mobile users, a big development process for the future could be offering the mobile version of the system for the other roles, teachers, school principal, etc. not only the parent and the student.

Another future development will be the mobile application not only on Android, but also on other operating systems as well, Windows Phone OS, iOS.

Could be considered also other roles for the application prevailed form real world activities in a school, as for example to create a virtual library with a new role, the librarian. This can be extended also to another subdomain which is secretariate.

The necessity of making scheduled backups and rollback procedures could be a good point to start for a further development, in order to create a more reliable system.

For the parents that use the mobile application the email notification has been already implemented , and instead of this, another more scalable solution could be based on push notifications technology integrated within the application.

Also if there is a long period of time that one user has not logged into the system, he could get a small preview of the recent discussions that take place on forums.

By increasing the security of sensitive data, there could be some ideas relying on the fact that it can be implemented a onetime login for each session with a onetime password that is send to the user via email or like a text message. Also when editing data that is sensitive, adding a new grade or motivating an absence, a face recognition algorithm could be beneficial when submitting the changes. Considering that the teacher has the system using his profile open and he must leave the system for something urgent, even if the system is open, nothing can be edited without the teacher involvement.

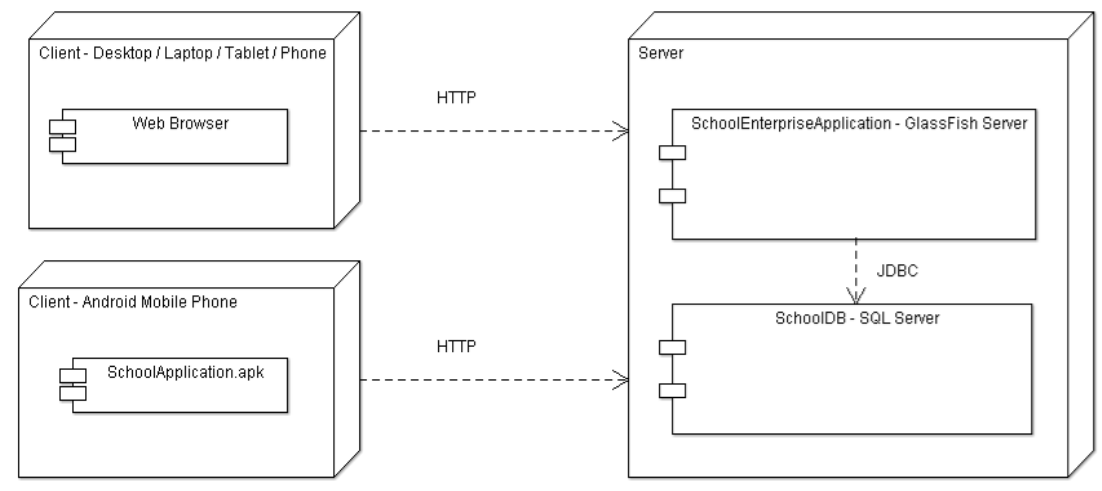

Fig 6: System deployment diagram 
Another more complex direction in further development could be complementing the actual GUI to be more appropriate for people with disabilities, like for example using color contrast to help highlight the accessible parts of the GUI.

\section{CONCLUSIONS}

The system manages to achieve the main goal, to be a competitive system with the existing solutions out there. The users get to be classified by roles, the management of documents including the school ledger, educational planning and schedule of classes was done successfully. The management of activities and also the evaluation of the students and teachers. The opportunity to have access to different types of data based on roles, to have the possibility to see statistics with the grades at school level or class level, etc..

The component that takes care of sending all the emails based on the asynchronous processing was implemented and works accordingly. The prediction component provides at this point good results even if we take into consideration that the algorithm has only a small amount of data to process. After more grades are collected from a large number of students the results offered by the prediction component will get more accurate and the component could really be a helpful one, to all undecided students.

The system can deliver an easy to learn space for the users and a very useful tool for the management of various school documents and activities.

\section{REFERENCES}

[1] Arora M. Deciphering phone and embedded security Part 1:Fundamentals of the Android architecture and terminologies, Available at: http://www.eetimes.com/document.asp?doc_id=1279698

[2] Biswas R., Ort E., The Java Persistance API - A Simpler Programming model for Entity persistence, Available at: http://www.oracle.com/technetwork/articles/java/jpa137156.html

[3] Edward J. 2014 .Mobile Applications Are Lilling The Free Web, Available at: http://www.businessinsider.com/mobile-web-vsapplication-usage-statistics-2014-4

[4] Han J., Kamber M., and Pie J. 2011. Data Mining Concepts and Techniques. 3rd edition, Morgan Kaufmann Publishers.

[5] Hansen D. 2007. SOA Using Java Web Services, Prentice Hall, 2007.
[6] Kalin M. 2013 .Java Web Services: Up and Running 2nd Edition, O'Reilly.

[7] Pandey M., V. Sharma V., A Decision Tree Algorithm Pertaining to the Student Performance Analysis and Prediction, International Journal of Computer Applications - Volume 61 - No. 13, Available at: http://citeseerx.ist.psu.edu/viewdoc/download?doi=10.1.1. 303.4290\&rep=rep1\&type $=$ pdf

[8] Witten E,. Frank E., Hall M., 2005 Data Mining: Practical Machine Learning Tools and Techniques 2rd Edition, Morgan Kaufmann Publishers.

[9] Characteristics of Message Driven Beans Available at: http://docs.oracle.com/javaee/6/tutorial/doc/gipko.html

[10] Comparing Primefaces, IceFaces and RichFaces with Google Trend, Available at http://liferayblogging.blogspot.com.tr/2014/01/comparing-primefacesicefaces-and.html

[11] Data Mining Concepts - About Classification, Available at

http://docs.oracle.com/cd/B28359_01/datamine.111/b281 29/classify.htm\#i1005746

[12] Documentation for https://javaserverfaces.java.net/docs/2.2/

[13] Documentation for PrimeFaces, http://primefaces.org/documentation.html

[14] Documentation for WEKA, http://weka.wikispaces.com/

[15] Enterprise JavaBeans, http://en.wikipedia.org/wiki/EJB

[16] Java Platform, Enterprise Edition, http://docs.oracle.com/javaee/7/index.html

[17] Password-Based Key Derivation Function 2, http://en.wikipedia.org/wiki/PBKDF2

[18] Salted Password Hashing , Available at : https://crackstation.net/hashing-security.htm

[19] Ekattor School Management System Pro , Available at:http://codecanyon.net/item/ekattor-school-managementsystem-pro/6087521

[20] Web documentation for Genymotion and download , Available at : https://www.genymotion.com/ 